\title{
A New CFD Model for VOC Emission Based on the General Adsorption Isotherm*
}

\author{
Baoqing DENG ${ }^{* *}$ and Chang Nyung $\mathrm{KIM}^{* * *}$
}

\begin{abstract}
This paper presents a new CFD model for the transportation of volatile organic compound (VOC) from building materials to the room air in the case with high VOC concentration in the air when Langmuir model or other nonlinear adsorption isotherms are used. In this model, the concentration equation solves the equivalent air-phase concentration and uses the equivalent air-phase diffusion coefficient to calculate the mass flux. A new parameter, specific mass, arising from adsorption isotherm is presented to account for the sorption and desorption effect. The present model and two previous models are applied to the steady and unsteady problems. Results show that the present model correctly simulates the emission of VOC from building materials. And the present model is easy to use with commercial CFD software.
\end{abstract}

Key Words: VOC Emission, CFD, Adsorption Isotherm

\section{Introduction}

In the last several decades, a variety of building and furnishing materials have been used in buildings. Thus, volatile organic compounds emitted from these materials have become the major source of indoor air pollution because of their large surface area and long-term existence. Since people stay in buildings for most of time, it is important to rank these materials so as to use low-emission materials in buildings as far as possible.

Although direct measurement on the chamber test and field study can provide accurate experimental data for VOC emission, it is costly and time-consuming. An alternate way of studying VOC emission is to use mathematical models. Among mathematical models, empirical models have been developed from experimental data by nonlinear fitting. However, they may not be scaled up for use in actual buildings. Thus, physical models have been more attractive to most researchers for modeling VOC emission, which are based on mass transfer mechanisms: diffusion within the material and surface emission at the materialair interface $e^{(1),(2)}$. For dry building materials, diffusioncontrolled models ignore the effect of surface emission

* Received 6th May, 2003 (No. 03-5061)

** Industrial Liaison Research Institute, Kyung Hee University, Yongin 449-701, Korea

*** College of Advanced Technology, Kyung Hee University, Yongin 449-701, Korea. E-mail: cnkim@khu.ac.kr since the internal diffusion is thought to be a major factor. As for wet materials, the effect of diffusion within the material is not taken into account in the emission models.

Therefore, some researchers turn to CFD model to study VOC emission from building materials. CFD model can simultaneously account for the diffusion of VOC in the material and the surface emission at the material-air interface. These models usually concern the following parameters: the diffusion coefficient in the material $D_{m}$, the material-air interface partition coefficient $K_{m a}$ or other adsorption isotherms and the initial concentration in the material $C_{m 0}$. In general, there are two kinds of CFD models: models based on the air-phase and solid-phase concentrations simultaneously and models based on only the equivalent air-phase concentration. For the former models, the diffusion coefficient in the material $D_{m}$ is used in the concentration equation. Since some experiments prefer to give the equivalent air-phase diffusion coefficient $D_{e}$ instead of $D_{m}$, there exists some inconvenience in the transformation of those two diffusion coefficients for some complicated adsorption isotherms ${ }^{(3)}$. By the way, the concentration is discontinuous because of the existence of adsorption isotherm, which means that this kind of model is not easy to be integrated into some commercial CFD software such as FLUENT because a continuous field is solved in such a software. Thus, many researchers have preferred to use the equivalent air-phase concentration so as to simplify the concentration equation ${ }^{(4)-(6)}$. This trans- 
formation means that a continuous dependent variable exists in the concentration equation. However, some problems may exist since enough consideration has not been given to the equivalent air-phase concentration and the diffusion coefficient adopted in the concentration equation, i.e., the inconsistency between the equivalent air-phase concentration and the diffusion coefficient in the concentration equation. Thus, the continuity of mass flux at the material-air interface does not seem to be smooth.

In the present study, a CFD model using the equivalent air-phase concentration is developed. In this model, the equivalent air-phase concentration is defined on the basis of the general adsorption isotherm. The equivalent air-phase diffusion coefficient depends on adsorption isotherm and the diffusion coefficient in the material. A new parameter, specific mass, is presented to account for the sorption effect. Through two cases, the difference between the present model and the previous models is depicted.

\section{Nomenclature}

$A:$ mass transfer area $\left(\mathrm{m}^{2}\right)$

$b:$ thickness of the material $(\mathrm{m})$

$C:$ Concentration $\left(\mathrm{kg} / \mathrm{m}^{3}\right)$

$C_{a d m 0}:$ maximum adsorption concentration $\left(\mathrm{kg} / \mathrm{m}^{3}\right)$

$C_{\text {in }}:$ inlet concentration

$C_{m 0}$ : material-phase initial concentration in the material $\left(\mathrm{kg} / \mathrm{m}^{3}\right)$

$c_{p}:$ specific heat at constant pressure $(\mathrm{J} /(\mathrm{kg} \cdot \mathrm{K}))$

$D:$ diffusion coefficient $\left(\mathrm{m}^{2} / \mathrm{s}\right)$

$D_{c}$ : effective diffusion coefficient $\left(\mathrm{m}^{2} / \mathrm{s}\right)$

$D_{e}$ : equivalent air-phase diffusion coefficient $\left(\mathrm{m}^{2} / \mathrm{s}\right)$

$f$ : function of general adsorption isotherm

$k$ : porosity of the material

$K_{m a}:$ material-air partition coefficient

$k_{l}$ : Langmuir model constant $\left(\mathrm{m}^{3} / \mathrm{kg}\right)$

$k_{e}:$ specific mass

$L:$ inlet and outlet width $(\mathrm{m})$

$p:$ mean pressure $(\mathrm{Pa})$

$T:$ temperature $(\mathrm{K})$

$t:$ time (s)

$\boldsymbol{u}_{j}:$ velocity $(\mathrm{m} / \mathrm{s})$

$V_{0}:$ inlet velocity $(\mathrm{m} / \mathrm{s})$

$V:$ volumetric air flow rate $\left(\mathrm{m}^{3} / \mathrm{s}\right)$

$\boldsymbol{x}_{j}$ : coordinates $(\mathrm{m})$

$y$ : coordinate in solid material $(\mathrm{m})$

greek symbols

$\lambda:$ thermal conductivity $(\mathrm{W} /(\mathrm{m} \cdot \mathrm{K}))$

$\lambda_{c}:$ mass conductivity $(\mathrm{kg} /(\mathrm{ms}))$

$\rho:$ density $\left(\mathrm{kg} / \mathrm{m}^{3}\right)$

subscripts

$a$ : air-phase

$i+:$ material side of the material-air interface

$i-$ : air side of the material-air interface $m$ : solid-phase

\section{Previous Models}

There are many CFD models investigating VOC emission. They can be categorized into the two kinds: the model based on the air-phase concentration and solidphase concentration and the model based on only the equivalent air-phase concentration. To clarify the problem, a review on two kinds of models will be given.

\subsection{Model A: based on the air-phase concentration} and solid-phase concentration ${ }^{(7)}$

Generally, it is assumed that the material is of homogeneous diffusivity. According to Fick's law, VOC transport within the material can be described by the following transient diffusion equation

$$
\frac{\partial C_{m}}{\partial t}=\frac{\partial}{\partial x_{j}}\left(D_{m} \frac{\partial C_{m}}{\partial x_{j}}\right)
$$

where $C_{m}$ is the solid-phase concentration of VOC in the material and $D_{m}$ the diffusion coefficient of VOC in the material. At the material-air interface there exists a concentration jump, meaning the concentration is discontinuous. This jump is usually described by adsorption isotherm at the material-air interface. It assumes the following form

$$
\left.C_{m}\right|_{i+}=f\left(\left.C_{a}\right|_{i-}\right)
$$

where $f$ means the function describing the general adsorption isotherm. There are several adsorption isotherms with respect to the characteristics of materials and VOCs. For low concentration of VOCs in the air, Henry's law is usually used

$$
\left.C_{m}\right|_{i+}=\left.K_{m a}\right|_{i-}
$$

where $K_{m a}$ is the material-air partition coefficient. For high concentration in the air, Langmuir model instead of Henry's law should be used.

$$
\left.C_{m}\right|_{i+}=\frac{\left.C_{a d m 0} k_{1} C_{a}\right|_{i-}}{1+\left.k_{1} C_{a}\right|_{i-}}
$$

VOC emitted from building materials enters into the air by emission at the material-air interface. The distribution of VOC in the room is determined by the convection of the air and molecular diffusion. For the case of turbulent flow, turbulent diffusion also plays an important role in VOC diffusion. In the present study, turbulent flow is not concerned. Thus, a transient convection-diffusion equation is used to describe the transport of VOC in the air

$$
\frac{\partial C_{a}}{\partial t}+\frac{\partial\left(u_{j} C_{a}\right)}{\partial x_{j}}=\frac{\partial}{\partial x_{j}}\left(D_{a} \frac{\partial C_{a}}{\partial x_{j}}\right)
$$

where $C_{a}$ is the air-phase concentration of VOC in the air, $D_{a}$ the diffusion coefficient of VOC in the air. Equations (1) and (5) imply the conservation of mass flux at 
any location of solution domain. Especially, it yields at the material-air interface

$$
-\left.D_{a} \frac{\partial C_{a}}{\partial x_{j}}\right|_{i-}=-\left.D_{m} \frac{\partial C_{m}}{\partial x_{j}}\right|_{i+}
$$

VOC emission is a conjugate mass transfer problem in nature. Therefore, VOC diffusion in the material and its transportation in the air should be solved simultaneously. The boundary conditions at the material-air interface consist of Eqs. (2) and (6). It should be pointed out that Eq. (2) must be given explicitly and Eq. (6) is implied by the conservation law at the material-air interface. Thus, the Model A cannot be solved directly by commercial CFD softwares, which solve a continuous dependent variable in the solution domain.

\subsection{Model B: based on the equivalent air-phase concentration $^{(4),(5)}$}

The Model A describes a discontinuous concentration field because of the explicit existence of Eq. (2). However, the conventional solver is based on the continuous dependent variable. Thus, the model based on only the equivalent air-phase concentration has been presented as follows;

$$
\begin{aligned}
& \frac{\partial C}{\partial t}=\frac{\partial}{\partial x_{j}}\left(D_{c} \frac{\partial C}{\partial x_{j}}\right) \\
& \frac{\partial C}{\partial t}+\frac{\partial\left(u_{j} C\right)}{\partial x_{j}}=\frac{\partial}{\partial x_{j}}\left(D_{a} \frac{\partial C}{\partial x_{j}}\right) \\
& -\left.D_{a} \frac{\partial C}{\partial x_{j}}\right|_{i-}=-\left.D_{c} \frac{\partial C}{\partial x_{j}}\right|_{i+}
\end{aligned}
$$

where $C$ is the equivalent air-phase concentration in the material and air, $D_{c}$ the effective diffusion coefficient of VOCs in the material, which is introduced by the following equation;

$$
D_{c}=\lambda_{c} /\left(k \rho_{a}+\rho_{m} \frac{d f(C)}{d C}\right)
$$

where $f$ is the function described by Eq. (2); $\lambda_{c}$ mass conductivity in the material and $k$ porosity of the material. The original Model B was written for porous media. For comparison, material porosity is not taken into account here. Since the Model B uses a continuous dependent variable in the concentration equation, it can be solved easily by commercial CFD software such as FLUENT. On the other hand, just like Eq. (6), Eq. (9) does not need to be given explicitly. It should be pointed out that how to define $D_{c}$ instead of $\lambda_{c}$ was given in the original Model B.

\section{New Model Development}

The above mathematical models for VOC emission have been applied to many problems. Compared with the Model B, the Model A has a complicated boundary condition at the material-air interface. Therefore, it is inconvenient for real buildings with many kinds of materials. Thus, the Model B is recommended for the simulation of
VOCs emission. Unfortunately, there exists the inconsistency between Eqs. (7) and (9), which will be shown later.

As we know, there exist a continuous diffusion flux and a concentration jump at the material-air interface. To eliminate the concentration jump from the mathematical model, the equivalent air-phase concentration should be introduced as follows;

$$
C= \begin{cases}f^{-1}\left(C_{m}\right) & \text { in the material } \\ C_{a} & \text { in the air }\end{cases}
$$

where $f$ is the function described by Eq. (2). Equation (11) transforms the air-phase and material-phase concentration into the equivalent air-phase concentration. It is known that the concentration is continuous within each region no matter what transformation is introduced. On the other hand, according to Eq. (2), there exists $\left.C\right|_{i+}=\left.C\right|_{i-}$, which means the equivalent air-phase concentration is continuous even at the material-air interface. Thus, the equivalent air-phase concentration is continuous in the whole domain. Meanwhile, using the equivalent air-phase concentration, Eq. (1) can be transformed into as follows;

$$
\frac{\partial(f(C))}{\partial t}=\frac{\partial}{\partial x_{j}}\left(D_{m} \frac{d f(C)}{d C} \frac{\partial C}{\partial x_{j}}\right)
$$

Here, two new parameters for the material are introduced to simplify Eq. (12): specific mass

$$
k_{e}=\frac{f(C)}{C}
$$

and the equivalent air-phase diffusion coefficient

$$
D_{e}=D_{m} \frac{d f(C)}{d C}
$$

Thus, Eq. (12) can be rewritten as

$$
\frac{\partial\left(k_{e} C\right)}{\partial t}=\frac{\partial}{\partial x_{j}}\left(D_{e} \frac{\partial C}{\partial x_{j}}\right)
$$

Equation (15) shows that the terms $D_{e}$ and $k_{e}$ represent the diffusion and accumulation of the equivalent airphase concentration. $D_{e}$ and $k_{e}$ have the same role of thermal conductivity and specific heat in the energy equation, respectively. $k_{e}$ means the actual change of amount of VOC when the equivalent air-phase concentration changes by a unit. On the other hand, Eq. (5) can be rewritten as follows;

$$
\frac{\partial C}{\partial t}+\frac{\partial\left(u_{j} C\right)}{\partial x_{j}}=\frac{\partial}{\partial x_{j}}\left(D_{a} \frac{\partial C}{\partial x_{j}}\right)
$$

The boundary condition, i.e., Eq. (6), is transformed into

$$
-\left.D_{a} \frac{\partial C}{\partial x_{j}}\right|_{i-}=-\left.D_{e} \frac{\partial C}{\partial x_{j}}\right|_{i+}
$$

Another boundary condition, i.e., Eq. (2), does not need to be given because of the use of the equivalent airphase concentration. Since the present model is deduced from the Model A by reversible transformation, the two models are equivalent in performance. Moreover, it has the same merit as the Model B has because it is also 
written for a continuous dependent variable. Although both the present model and the Model B solve the equivalent air-phase concentration in the concentration equation, Eqs. (15) and (17) are different from Eqs. (7) and (9), respectively. And the two models cannot be transformed into each other. The further study proves that there exists a built-in inconsistency between Eqs. (7) and (9). In fact, Eq. (15) is a conservative equation. The non-conservative equation can be also written as follows;

$$
k_{e}^{\prime} \frac{\partial C}{\partial t}=\frac{\partial}{\partial x_{j}}\left(D_{e} \frac{\partial C}{\partial x_{j}}\right)
$$

with

$$
k_{e}^{\prime}=\frac{d f(C)}{d C}
$$

In the derivation of the Model B, Eq. (7) was obtained by dividing $k_{e}^{\prime}$ on the two sides of Eq. (18) and $D_{c}=D_{e} / k_{e}^{\prime}=$ $D_{m}$ was set correspondingly. Obviously, this simplification is valid only when $k_{e}^{\prime}$ is a constant. Henry's law strictly meets this requirement and Langmuir model cannot satisfy it well with high concentration. Thus, some error would be introduced in the use of Langmuir model. On the other hand, after introducing this simplification, $D_{c} \frac{\partial C}{\partial x_{j}}$ is not the true mass flux, just like $\frac{\lambda}{c_{p}} \frac{\partial T}{\partial x_{j}}$ is not the true heat flux. Since $D_{a} \frac{\partial C}{\partial x_{j}}$ is the true mass flux all along, the boundary condition in the Model B, i.e., Eq. (9), is inappropriate and valid only when $k_{e}^{\prime}=1$ exists, which means that the application of Eq. (9) would introduce much error. It can be concluded that the Model B must underestimate the concentration of VOC in the room air since the mass resistance in the solid material is exaggerated. And the concentration in the solid material calculated from the Model B would decay faster than those of the other two models because of the absence of specific mass. On the other hand, if $D_{c}=D_{e}$ is set, it seems that the conservation of mass flux at the material-air interface is satisfied by Eq. (9). As a matter of fact, it leads to that VOC concentration in the air would increase faster in the early stage and decrease faster in the decay stage than the real case because $D_{e}$ is much bigger than $D_{m}$ and Eq. (7) violates Eq. (18). Thus, no diffusion coefficient can meet Eqs. (7) and (9) simultaneously unless $k_{e}^{\prime} \equiv 1$ exists, which leads to the inconsistency between Eqs. (7) and (9) for the general adsorption isotherm.

\section{Results and Discussion}

Scenario 1: steady mass transfer of VOC

Figure 1 depicts the geometry configuration studied. The solid material separates two chambers. The chamber A has a constant supply of polluted air while the chamber $B$ is ventilated by clean air. N-octane diffuses from the chamber A into the chamber B through the material and arrives at steady state at last. Air exchange rate in both

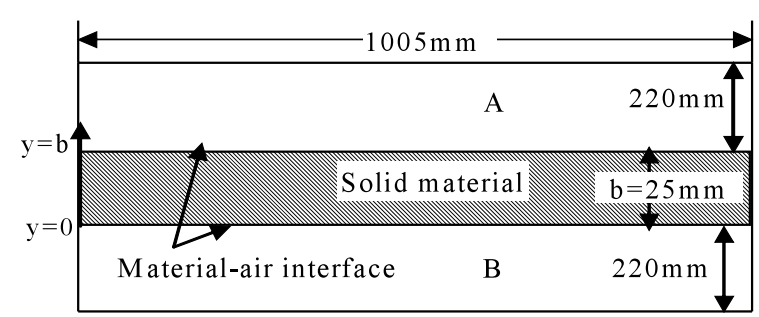

Fig. 1 Geometry for mass transfer through a solid material between two chambers

Table 1 Parameters for steady state calculation

\begin{tabular}{|c|c|c|c|c|c|}
\hline & $\mathrm{D}_{\mathrm{e}}\left(\mathrm{m}^{2} / \mathrm{s}\right)$ & $\mathrm{D}_{\mathrm{a}}\left(\mathrm{m}^{2} / \mathrm{s}\right)$ & $\mathrm{D}_{\mathrm{c}}\left(\mathrm{m}^{2} / \mathrm{s}\right)$ & $\mathrm{K}_{\mathrm{ma}}$ & $\mathrm{k}_{\mathrm{e}}$ \\
\hline Brick wall & $3.25 \times 10^{-7}$ & $6.0 \times 10^{-6}$ & $1.48 \times 10^{-8}$ & 21.9 & 21.9 \\
\hline
\end{tabular}

chambers is set at $2 \mathrm{~h}^{-1}$. A fan in each chamber is placed for air flow. Inlet concentrations for the chamber $\mathrm{A}$ and B are $11 \mathrm{mg} / \mathrm{m}^{3}$ and zero, respectively. Model parameters are shown in Table 1, which are taken from Meininghaus et al. ${ }^{(3)}$

This geometry was used to measure the equivalent air-phase diffusion coefficient of VOC in the material and to investigate the sorption effect of the material. Meininghaus et al. measured the outlet concentration of the chamber B with time and calculated the material-air interface partition coefficient and the equivalent air-phase diffusion coefficient of VOC in the material ${ }^{(3)}$.

There are two ways to solve this problem. The one is to solve one-dimensional mass transfer problem through the solid material, which was taken by Meininghaus, et al. ${ }^{(3)}$ For this method, the concentrations in each chamber are thought to be uniform and the governing equation can be written as;

$$
\frac{d}{d y}\left(D \frac{d C}{d y}\right)=0
$$

where $y$ is the coordinate normal to the material-air interface as shown in Fig. 1 and $D$ takes the value of $D_{e}$ or $D_{c}$, which depends on the model used. The boundary conditions are written as follows;

$$
\begin{aligned}
& -A D \frac{d C}{d y}=V\left(C-C_{\text {in }}\right) \quad \text { at } \quad y=b \\
& A D \frac{d C}{d y}=V C \quad \text { at } \quad y=0
\end{aligned}
$$

Its solution is

$$
C=\frac{C_{\text {in }}}{2 A+V b / D}\left(\frac{V}{D} y+A\right)
$$

The other one is to solve three-dimensional convection-diffusion equation using CFD. No-slip condition is used at all walls and no concentration gradient exists at all walls but the material-air interface. In the present study, FLUENT 6 is used to perform numerical calculation. The one-dimensional and three-dimensional 
numerical solutions yield Table 2 showing the outlet concentration of the chamber B. Result from the present model agrees well with the experimental value and result from the Model B seriously underestimates the outlet concentration in the chamber B. There exists a little discrepancy between one-dimensional and threedimensional results. And the former is more close to the experimental value than the latter because the equivalent air-phase diffusion coefficient is evaluated on the basis of one-dimensional model.

Scenario 2: unsteady VOC emission

Scenario 1 depicts the influence of different models on steady mass transfer. In this section, an unsteady VOC emission will be investigated to show the influence of different models on unsteady mass transfer. A twodimensional room with size of $4.5 \mathrm{~m} \times 3 \mathrm{~m}$ is used as shown in Fig. 2. The widths of both inlet and outlet are $0.02 \mathrm{~m}$. There exists a dry material of $0.005 \mathrm{~m}$ thickness on the bottom floor. VOC emits from the dry material and diffuses into the air. The fresh air flows into the room through the inlet at a speed of $0.1 \mathrm{~m} / \mathrm{s}$ and goes out through the outlet.

Two adsorption isotherms are used to investigate the emission for the three CFD models. All parameters are shown in Table 3, taken from Yang et al. and Benkhedda et al. ${ }^{(7),(8)}$ FLUENT 6 is used to solve this two-dimensional problem with three models. Since FLUENT cannot treat the boundary condition of the Model A directly, a C language subroutine should be written to meet the requirement.

Figure 3 depicts the average concentration in the

Table 2 Outlet concentration of the chamber B

\begin{tabular}{|l|c|}
\hline & Concentration $\left(\mathrm{mg} / \mathrm{m}^{3}\right)$ \\
\hline Present model with Eq. (23) & 1.03 \\
\hline Present model: three-dimensional & 0.98 \\
\hline the Model B with Eq. (23) & 0.057 \\
\hline the Model B: three-dimensional & 0.05 \\
\hline Experimental value from Meininghaus et al. [3] & 1.03 \\
\hline
\end{tabular}

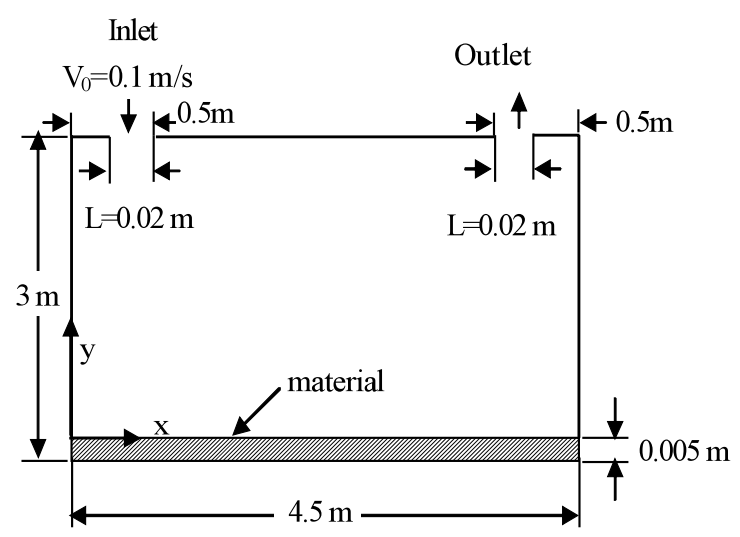

Fig. 2 Room model room with time. No matter what type of adsorption isotherm is used, the present model obtains the same result as the Model A all along. However, the Model B seriously underestimates the average concentration in the room compared with the other two models because the Model B exaggerates the mass transfer resistance in the solid material. This shows that the boundary condition in the Model B, i.e., Eq. (9), is wrong. Moreover, since no specific mass exists in the Model $\mathrm{B}$, the computational concentration in the solid material decays faster than the

Table 3 Model parameters

\begin{tabular}{|c|c|}
\hline Initial concentration & $\mathrm{C}_{\mathrm{m} 0}=5.28 \times 10^{-2} \mathrm{~kg} / \mathrm{m}^{3}$ \\
\hline Henry's law & $\mathrm{K}_{\mathrm{ma}}=3289$ \\
\hline Langmuir model & $\mathrm{C}_{\mathrm{adm} 0}=333 \mathrm{~kg} / \mathrm{m}^{3}, \mathrm{k}_{1}=1.24 \mathrm{~m}^{3} / \mathrm{kg}$ \\
\hline $\mathrm{D}_{\mathrm{c}}$ & $7.65 \times 10^{-11} \mathrm{~m}^{2} / \mathrm{s}$ \\
\hline $\mathrm{D}_{\mathrm{m}}$ & $7.65 \times 10^{-11} \mathrm{~m}^{2} / \mathrm{s}$ \\
\hline
\end{tabular}

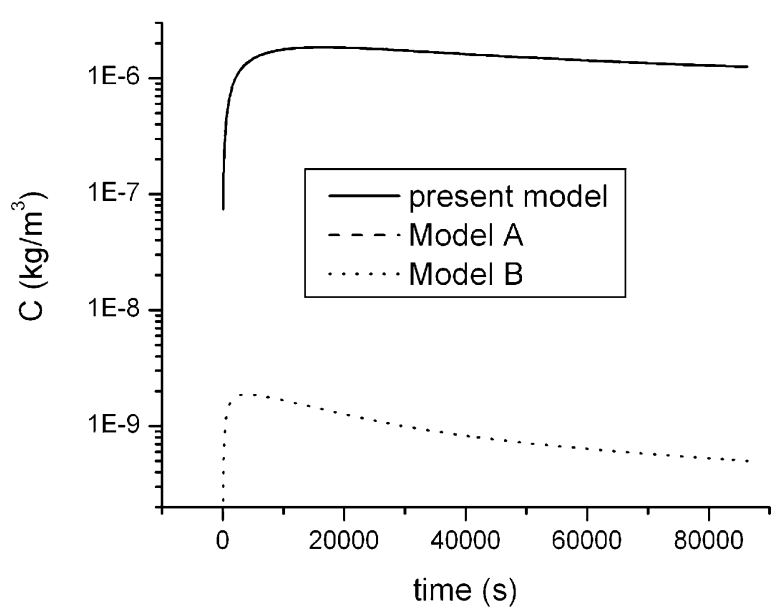

(a)

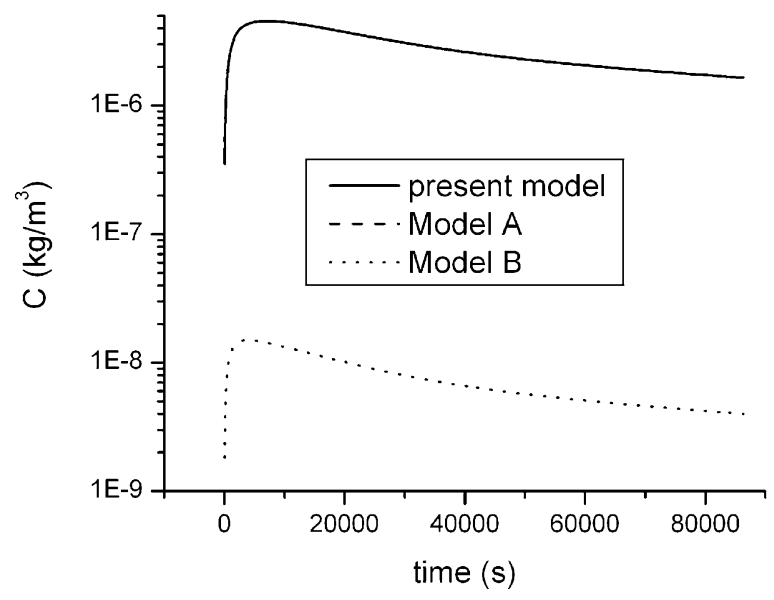

(b)

Fig. 3 The average concentration in the room with time (lines for the present model and the Model A coincide each other in each graph), (a) Henry's law, (b) Langmuir model 
true one. For Henry's law, the percentages of the concentration in the material at $24 \mathrm{~h}$ to the initial concentration are $71 \%$ for the present model, $71 \%$ for the Model A and $42 \%$ for the Model B, respectively.

In the case of Langmuir model, Figs. 4 and 5 depict the specific mass and the equivalent air-phase diffusion coefficient in the material with time, each of which keeps a constant, meaning $k_{e}=C_{a d m 0} k_{1}$ and $D_{e}=C_{a d m 0} k_{1} D_{m}$, respectively. The reason lies in that $C_{m 0}=5.28 \times 10^{-2} \mathrm{~kg} / \mathrm{m}^{3}$, which is taken from Yang et al. [2001], is too small relatively to the maximum adsorption concentration $C_{a d m 0}$ and thus Langmuir model reduces to Henry's law. Although the specific mass and the equivalent air-phase diffusion coefficient in the material keep a constant in this case, they are actually the nonlinear functions of the local concentration while the latter depends on the spatial coordinate and time. The case of $C_{m 0}=5.28 \times 10^{-2} \mathrm{~kg} / \mathrm{m}^{3}$ is only a special example at low concentration.

Also in Figs. 4 and 5 shown is each of the two curves

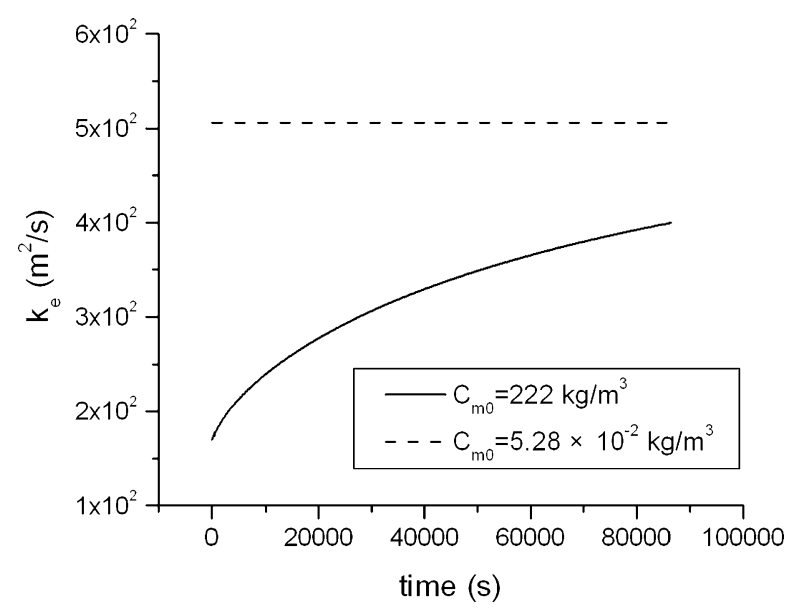

Fig. 4 The volume-averaged specific mass with time

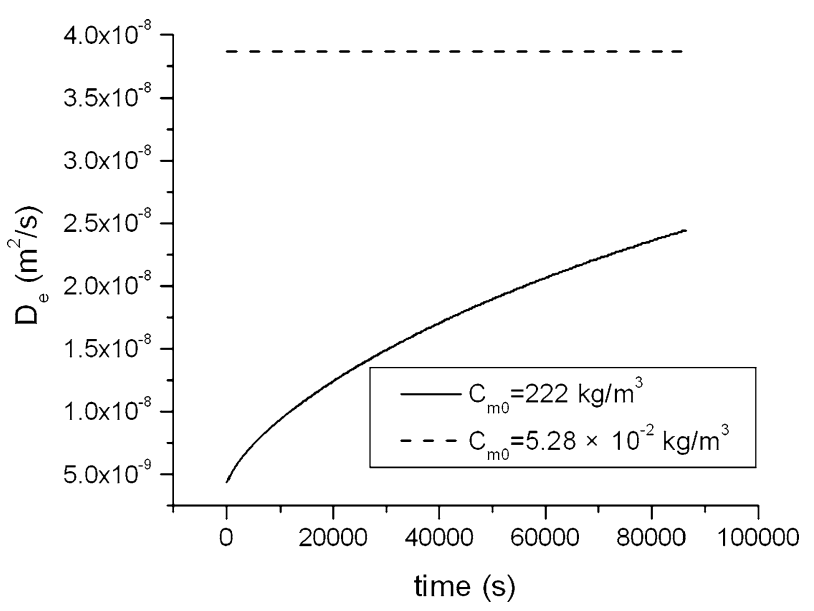

Fig. 5 The volume-averaged equivalent air-phase diffusion coefficient with time corresponding to $C_{m 0}=222 \mathrm{~kg} / \mathrm{m}^{3}$, which means the two thirds of the maximum adsorption concentration $C_{a d m 0}$, while the ratio of the initial concentration to the maximum adsorption amount is 0.64 in the study by the original Model $\mathrm{B}^{(4),(5)}$. Each of the two variables is small at the beginning because of high concentration in the material and increases with time. With the decrease of the concentration in the material, each of the two variables tends to be close to the corresponding value of low initial concentration, i.e., a constant. Meanwhile, $k_{e}$ and $k_{e}^{\prime}$ are the function of spatial coordinate at high concentration for the general adsorption isotherm, as shown in Fig. 6. Thus, Eq. (7) must introduce some error because of the elimination of $k_{e}^{\prime}$ when it is compared with Eq. (18). It can be found that the specific mass is lower if the concentration is higher. Moreover, $k_{e}$ is bigger than $k_{e}^{\prime}$ all along.

Figure 7 shows the concentration contour in the room at $24 \mathrm{~h}$. Since the air flow pattern is identical, the concentration distributions for different models are expected to be similar. However, the concentration from the Model B is much smaller than that from the present model and the Model A. Compared with the Model A, the present model is useful and can be easily integrated into the commercial CFD software because the conjugate mass transfer method is available.

\section{Conclusion}

The equivalent air-phase concentration simplifies the numerical simulation of VOC emission from the solid material to the air with a concentration jump at the interface. However, the boundary condition at the material-air interface must be treated carefully to ensure the conservation law of mass there. In this paper, we have presented a new CFD model based on the equivalent air-phase concentration. Moreover, the equivalent air-phase diffusion coefficient has also been introduced to ensure the conservation law of mass. A new parameter, specific mass, arising

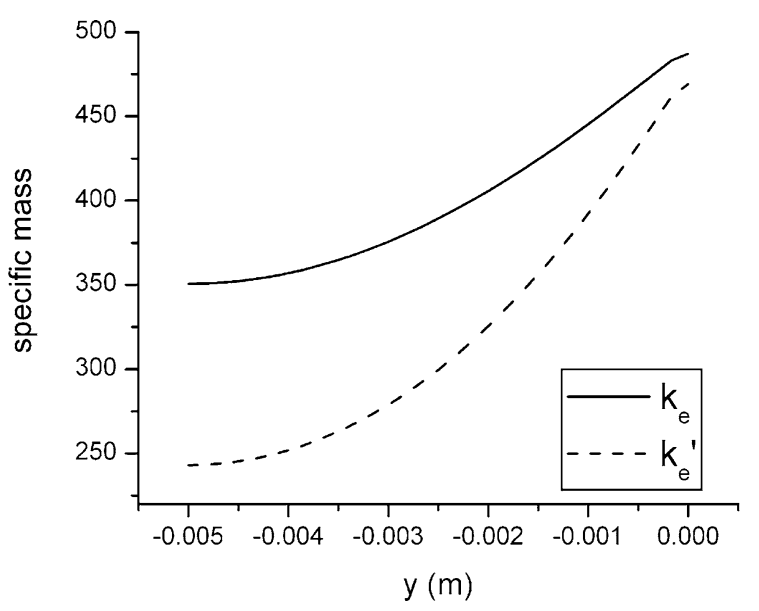

Fig. 6 Specific mass at $x=2.25 \mathrm{~m}$ with $C_{m 0}=222 \mathrm{~kg} / \mathrm{m}^{3}$ 


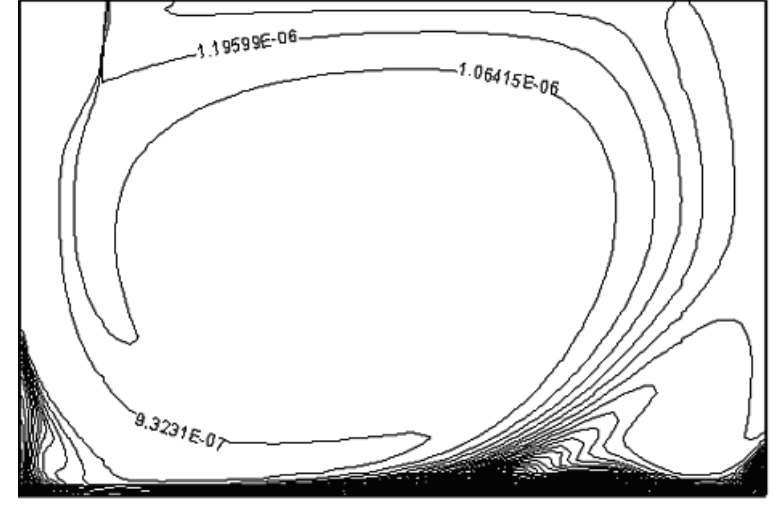

(a)

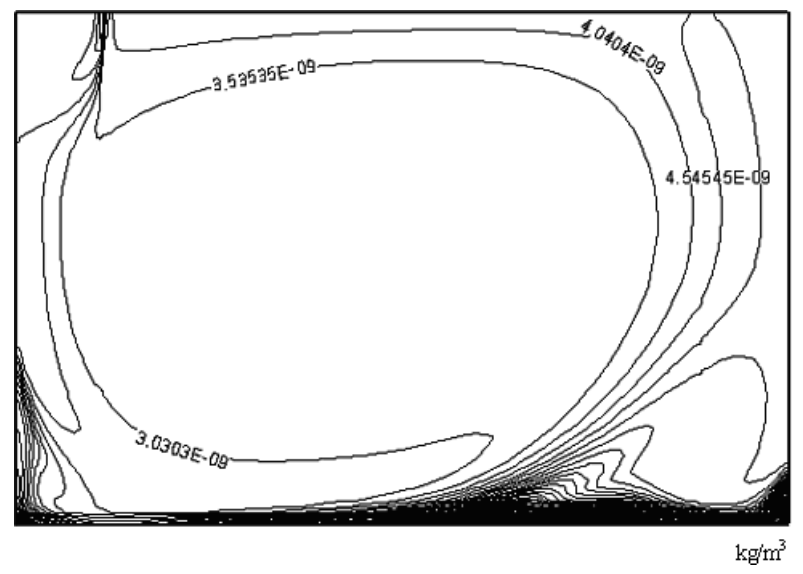

(b)

Fig. 7 Concentration distributions in the room at $24 \mathrm{~h}$, (a) The present model and the Model A, (b) The Model B

from adsorption isotherm is presented to account for the sorption effect. The present model and the two previous models are applied to both steady and unsteady problems. Result of the present model on the steady problem agrees well with the experimental value. And the calculation results on the unsteady problem with the present model also agree well with that of the Model A and are different from that of the Model B. Compared with the Model A, the present model is easy to use with commercial CFD software in the case with high VOC concentration in the air where Langmuir model is to be used.

\section{Acknowledgements}

This work was supported by Korea Research Foundation Grants (KRF-2001-005-E0004).

\section{References}

( 1 ) Little, J.C., Hodgson, A.T. and Gadgil, A.J., Atmospheric Environment, Vol.28 (1994), pp.227-234.

( 2 ) Sparks, L.E., Tichenor, B.A. and Chang, J., et al., Indoor Air, Vol.6 (1996), pp.31-40.

(3) Meininghaus, R., Gunnarsen, L. and Knudsen, S.N., Environmental Science and Technology, Vol.34 (2000), pp.3101-3108.

(4) Murakami, S., Kato, S., Ito, K., et al., ASHRAE Transaction (Winter Meeting in Atlanta), AT-01-13-3, (2001).

( 5 ) Murakami, S., Kato, S., Ito, K. and Zhu, Q., Indoor Air, Vol.13(s6) (2003), pp.20-27.

( 6 ) Yang, X., Chen, Q. and Bluyssen, P.M., ASHRAE Transactions, Vol.104 (1998), pp.1297-1308.

( 7 ) Yang, X., Chen, Q. and Zhang, J.S., et al., Building \& Environment, Vol.36 (2001), pp.1099-1107.

( 8 ) Benkhedda, J., Jaubert, J., Barth, J., Perrin, L. and Bailly, M., The Journal of Chemical Thermodynamics, Vol.32 (2000), pp.401-411. 